\title{
RIESGO Y REESTRUCTURACIÓN ESPACIAL EN LA CUENCA URBANA DEL RÍO TUNJUELO Aportes para el estudio de una espacialidad del riesgo
}

\author{
Leonel Adrián Cárdenas Roa \\ Universidad Nacional de Colombia, Maestría en Urbanismo \\ Director de la investigación: Luis Carlos Jiménez Mantilla \\ E-Mail: ad_cardenas@yahoo.com
}

\section{RESUMEN}

El posicionamiento técnico y político de la categoría de riesgo en las últimas tres décadas en Colombia y a nivel global es uno de los factores que está definiendo el proceso de reestructuración espacial actualmente en curso en la ciudad de Bogotá. El estudio de la cuenca urbana del río Tunjuelo permite contar con una mirada global de la evolución de este proceso, a partir del cual se ha configurado una espacialidad específica, diferenciada y contingente frente a las visiones urbanas sobre las que se ha buscado estructurar la ciudad desde finales del siglo XX. Este trabajo se enmarca así en los debates actuales frente al urbanismo y el ordenamiento territorial, en los que los discursos del riesgo, la resiliencia o la adaptación al cambio climático se han convertido en elementos centrales de la discusión en torno al futuro de las ciudades.

Palabras clave: urbanismo, riesgos urbanos, producción del espacio.

\begin{abstract}
The technical and political positioning of the risk category in the past three decades in Colombia and globally is one of the factors that is defining the spatial restructuring process that is currently occurring in the city of Bogota. The study of the urban area of the Tunjuelo river watershed gives a global view of the evolution of this process, in which there are characteristics of specific, differentiated and contingent spatiality, in comparison to late 20th century urban visions that were intended to structure the city. This study is framed within contemporary debates on urbanism and land-use planning, where themes of risk, resilience and climate change adaption have become central discussion issues around the future planning of cities.
\end{abstract}

Key words: urbanism, urban risk, production of urban space. 


\section{A PROPÓSITO DE LA INVESTIGACIÓN}

Existen en Bogotá una serie de intervenciones físicas asociadas a la gestión del riesgo que están reconfigurando las estructuras urbanas de los barrios de origen informal, produciendo un cambio evidente y significativo en el tejido social y espacial de la ciudad. La indagación frente al estado actual de este fenómeno y la revisión de sus orígenes y evolución permitieron realizar una aproximación al estudio de los procesos urbanos contemporáneos bajo el enfoque del riesgo, entendido como concepto clave en la formación de la sociedad y la ciudad contemporánea (Cárdenas Roa, 2016). En este sentido, lo que más llamó la atención de las transformaciones urbanas observadas al momento de formular la investigación fue la ruptura con la linealidad de los procesos de urbanización, lo que llevó a formular un recorrido por las ideas y los procesos urbanos asociados al riesgo y su rol frente a las dinámicas de reestructuración espacial actualmente en curso en la ciudad.

De esta forma, la pregunta de investigación planteó cómo la adopción y evolución de la categoría de riesgo a nivel institucional ha incidido en la producción y reestructuración del espacio urbano en Bogotá. Para responder a esta pregunta se formuló como objetivo general establecer las relaciones existentes entre el riesgo y los procesos de reestructuración espacial actualmente en curso en la cuenca urbana del río Tunjuelo. Como objetivos específicos se plantearon: a) establecer los fundamentos teóricos y conceptuales de la categoría del riesgo en su relación con el espacio, los fenómenos urbanos y el urbanismo contemporáneos, b) reconocer la evolución y el alcance de la política institucional en torno al riesgo desde una perspectiva territorial y urbana en Colombia, y c) identificar las formas espaciales asociadas al riesgo producto de las respuestas y estrategias adoptadas por la administración distrital en el espacio urbano de la cuenca del río Tunjuelo.

Es de resaltar la confluencia en la investigación de las corrientes de pensamiento europeo y latinoamericano en torno al riesgo y su desarrollo particular en el urbanismo y los estudios urbanos. Si el enfoque europeo permite cuestionar el monopolio de la toma de decisiones que se presentan como técnicas pero cuyo alcance final resulta social y político, el enfoque latinoamericano, orientado fundamentalmente a la dimensión material del riesgo, aporta las variables de la vulnerabilidad y la desigualdad para el estudio crítico de los desastres.

Se pueden resaltar así en el pensamiento europeo el trabajo de Beck en torno a la sociedad del riesgo y la modernización reflexiva $(1998,2002)$ y las elaboraciones que en este sentido desarrollan Ascher (2004) y Borja $(2009,2013)$ a propósito de un urbanismo reflexivo. Por su parte, en la perspectiva latinoamericana son de referencia los estudios iniciados por La RED (1993,1996a, 1996b) en los que se destacan las relaciones entre ciudad, riesgo y desastres. Pero es fundamentalmente en la última década, a partir de la realización de investigaciones empíricas orientadas a establecer las relaciones entre riesgo y urbanización en el contexto de la ciudad latinoamericana, que se ha venido presentando una convergencia conceptual entre ambas corrientes (D'Ercole, Hardy, Metzger, \& Jérémy, 2009; Lampis, 2013; Lampis \& Rubiano, 2012; Rebotier, 2009, 2014; Zeiderman, 2012).

En este sentido, la hipótesis formulada para el estudio de la cuenca urbana del río Tunjuelo planteó la emergencia de una espacialidad del riesgo que es necesario reconocer e incorporar a la disciplina del urbanismo, la cual responde a las relaciones desiguales de riesgo bajo las cuales se ha producido y se sigue produciendo la ciudad, en un contexto socioespacial con altos niveles de injusticia y exclusión social. Si por una parte el uso generalizado de esta categoría ha derivado en la producción de discursos y agendas urbanas globales y genéricas en torno a la resiliencia y la adaptación al cambio climático, no se puede desconocer que la aplicación creciente de las políticas asociadas al riesgo está produciendo una espacialidad específica, diferenciada y contingente que pone en evidencia las historias, contextos y desarrollos particulares de nuestras ciudades.

\subsection{El estudio de caso}

La metodología para el estudio de caso implicó realizar un análisis de la cuenca urbana del río Tunjuelo en dos marcos temporales: 1 . Un recorrido histórico que dio cuenta del proceso de estructuración urbana de la cuenca durante el siglo XX, y 2. Un ejercicio de investigación en torno al registro de daños y pérdidas, la exposición a amenazas naturales y los planes, programas y proyectos urbanos asociados, permitiendo identificar las transformaciones de las estructuras socioespaciales del área de estudio en los últimos 15 años y su correspondencia con la estrategia territorial elaborada a inicio de la década del 2000 en el marco 
de la formulación del Plan de Ordenamiento Territorial -POT- de Bogotá. Para esto, se recurrió a las siguientes fuentes cartográficas y bases de datos para el análisis de los procesos urbanos en la cuenca y la ciudad:

Cartografía: el análisis cartográfico realizado se orientó a la realización de dos ejercicios prácticos. Una caracterización espacial de la cuenca del río Tunjuelo y un análisis histórico de su desarrollo urbano, para lo que se recurrió al uso de herramientas digitales SIG. Las bases de datos espaciales consultadas y procesadas para este ejercicio fueron el Marco Geoestadístico Nacional del DANE (2012) y el Mapa de Referencia de la IDECA (junio de 2015). Adicionalmente, se recurrió a otras fuentes cartográficas que requirieron ser digitalizadas o que fueron utilizadas para contrastar y completar la información obtenida de las bases de datos espaciales consultadas, entre estas: la cartografía "POMCA del río Bogotá" de la CAR (2006), el documento "Diagnóstico de la Cuenca Hidrográfica del río Tunjuelo" de la CAR (2007), la cartografía IDIGER (2015) y la cartografía SINUPOT (2015). Para el análisis histórico del desarrollo urbano de la cuenca se recurrió a digitalizar, sobre la base del Mapa de Referencia de la IDECA (junio de 2015), la cartografía "Formas de crecimiento urbano" de la Universidad Nacional ubicada en el Hall del edificio Sindu, cuyo registro llega hasta el año 1984. Este análisis se completó con un corte parcial a partir análisis del Servicio WMS Ortofoto de la ciudad de Bogotá, del Año 1998.

Bases de datos: el análisis de las bases de datos se orientó a la realización de tres ejercicios prácticos: 1 . Caracterizar la población, áreas y densidades en la cuenca y la ciudad en la actualidad, 2. Analizar los daños y pérdidas ocurridos en la cuenca y la ciudad desde el año 2002 y de la evolución de la exposición a amenazas desde el año 2006, y 3 . Sistematizar y analizar los programas, planes y proyectos de inversión de la ciudad en torno al riesgo desde el año de 1998. Para el análisis de datos poblacionales y de áreas, se recurrió a la consulta del Mapa de Referencia de la IDECA (junio de 2015), Inventario Estadístico de la SDP (2012) y Series de Población 1985-2020 del DANE. Para el análisis de daños y pérdidas y la exposición a amenazas naturales se recurrió a la consulta de la base de datos del Observatorio Distrital de Gestión del Riesgo -ODGR-CC-. Por último, para el análisis de los programas, planes y proyectos de inversión se recurrió a consultar y sistematizar las Memorias de los Planes de Desarrollo Distritales de la SDP en los componentes asociados al riesgo, vivienda, infraestructura básica y desarrollos informales.

\section{UNA APROXIMACIÓN CONCEPTUAL A LA RELACIÓN ENTRE RIESGO, URBANISMO Y URBANIZACIÓN}

\subsection{Riesgo y sociedad global}

La teoría elaborada por Beck se formuló originalmente para explicar el cambio social y la crisis del paradigma moderno iniciado desde la década de 1970 en países con fuerzas productivas altamente desarrolladas, aunque su elaboración posterior ha expuesto el alcance global de los procesos sociales asociados al riesgo (Figura 1). Desde esta perspectiva, el riesgo se define como un dispositivo de racionalización del azar para reducir la indeterminación, por lo cual se encuentra asociado a la contingencia y a la forma en como ésta es afrontada socialmente (Beriain, 2007).

Una de las claves para entender estas transformaciones se encuentra en la relación entre presente y futuro. La sociedad moderna, al tener como uno de sus fundamentos la ampliación de las opciones y la libertad de elección frente al futuro, necesariamente expande los riesgos, con lo que aumenta la posibilidad de que ocurra lo no esperado, lo contingente. La idea de un futuro controlado desde las acciones presentes por medio del ejercicio de la planificación y el diseño y soportado sobre el paradigma del desarrollo moderno, ha dado paso a otro futuro, en el que la sociedad debe asumir como propios los efectos retroactivos y no deseados del progreso. Esto significa, según Beck, la posibilidad de ampliar el campo democrático hacia la toma de decisiones que se han presentado comúnmente como un problema de competencia técnica, pero que en su desarrollo y resultados tienen impactos ecológicos, sociales y políticos de interés colectivo (Beck, 1998; Beriain, 2007). 




Figura 1. Sociedad industrial vs. Sociedad del riesgo (Ulrick Beck).

Fuente: elaboración propia.

Esta nueva situación abre la posibilidad de cuestionar las decisiones que se toman en nombre del progreso ya que provocan potenciales efectos secundarios a nivel social y ambiental. La sociedad del riesgo sería así una sociedad en proceso de realización, producto del triunfo de la sociedad industrial semimoderna. Su característica se expresa en la ruptura con las fronteras e instituciones tradicionalizadas por la sociedad industrial, redefiniendo los límites y perspectivas frente al futuro. Pero paradójicamente, si bien los riesgos y sus efectos tienen una tendencia a desencadenar procesos de alcance global (Beck, 2002), en su dimensión material su producción y distribución es geográfica y socialmente desigual (García Acosta, 2005), consolidándose como un fenómeno glocal (Figura 2).



Figura 2. Enfoques frente a la construcción social del riesgo.

Fuente: elaboración propia.

\subsection{Riesgo y urbanización. La configuración de una espacialidad del riesgo}

Si bien la sociedad del riesgo de Beck constituye una visión optimista frente a la posibilidad de una sociedad global con capacidad de superar el destino de peligro y las situaciones provocadas por el paradigma de desarrollo moderno, las fuerzas capitalistas han logrado aprovechar económica y políticamente los riesgos, elevando a un nuevo nivel la lógica del desarrollo moderno. En este escenario no se eliminan los riesgos, sino que se niegan, acumulan o se reproducen ante su potencial económico o la amenaza que puede representar su reconocimiento en distintos nichos de mercado (Beck, 2002). Se configura así lo que se podría denominar, desde una visión fatalista, la idea de una sociedad del desastre, en la que la incertidumbre y el clima de miedo dominan los procesos sociales y económicos. 
Autores como Sassen (2015) y Davis (2007) exponen esta nueva dinámica del capital y dan cuenta de los procesos de agudización de las desigualdades, concentración de la riqueza y generación de nuevas espacialidades urbanas de las últimas décadas a nivel global. Se trata de espacialidades en las que se convive, antes que con la conciencia del riesgo, con la situación de desastre, donde se han normalizado las relaciones sociales de inequidad y poder y donde se ha naturalizado la idea de catástrofe.

De manera alternativa, la lectura de Ascher (2004) frente a la ciudad contemporánea plantea una aproximación dialéctica que articula conceptualmente los procesos sociales asociados al riesgo y la dinámica de urbanización global. Si por una parte la idea de crisis urbana hace referencia a una situación excepcional que al ser superada supone un retorno a un estado normal de las cosas, la idea de riesgo implica una situación de normalidad a superar que se convierte en el motor para avanzar a nuevas formas sociales y políticas (Figura 3). Esta perspectiva permite articular las formas sociales asociadas al riesgo y la modernización reflexiva con la concepción lefebvriana de la revolución urbana (Ascher, 2004).



Figura 3. Revolución urbana vs. Crisis urbana.

Fuente: elaboración propia.

En este sentido, la revolución urbana se puede definir como un proceso dual. Crea, por una parte, "nuevas actitudes frente al futuro, nuevos proyectos, formas de pensar y de actuar diferentes" (Ascher, 2004, p. 55), produce un "cambio de escala, de forma de gobierno, de base socioeconómica y de nuevos comportamientos y valores colectivos" (Borja, 2013, p. 9). Simultáneamente, estos cambios se acompañan de la agudización de las desigualdades y los procesos de acumulación, en donde grandes grupos de población son expulsados de los sistemas sociales, económicos y biosféricos, producto de una concepción dominante y estrecha de crecimiento económico (Sassen, 2015). En esta espacialidad son particularmente los colectivos marginados y expulsados en el proceso de revolución urbana los que asumen de manera desigual las consecuencias de la producción, acumulación y distribución de riesgos de la sociedad moderna, a manera de expropiaciones espaciales, ecológicas y económicas, configurando, lo que se denomina en este trabajo como una espacialidad del riesgo.

\subsection{Riesgo, incertidumbre y urbanismo}

La convergencia de las nuevas relaciones de riesgo y el proceso de urbanización global plantea nuevos problemas al urbanismo. Desde finales del siglo XX la respuesta a este escenario ha comprendido las dos figuras procedimentales del urbanismo definidas por Choay (1997): las reglas y los modelos. Por una parte, ha significado la formulación y difusión de nuevos modelos urbanos asociados a la institucionalización del riesgo, mediante el posicionamiento de las figuras de la ciudad sostenible y la ciudad "resiliente". Por otra parte, ha exigido la redefinición de los sistemas de reglas y principios sobre los que se ha organizado la planificación y el urbanismo moderno, ante la necesidad de enfrentar las contingencias del desarrollo dentro de un marco de riesgos e incertidumbre cada vez más amplio.

\subsubsection{El riesgo en los modelos urbanos: la ciudad sostenible y la ciudad resiliente}

El fenómeno del cambio climático ha llevado a la necesidad de integrar la gestión ambiental con la gestión del riesgo de desastres (Lavell, 2010). En este proceso de integración se han superpuestos los discursos urbanos de la ciudad sostenible, de mayor arraigo temporal, con los de la ciudad resiliente, como figura emergente. A estos discursos les ha correspondido el modelo físico-espacial de la ciudad compacta, una aproximación que suele presentarse como técnica y objetiva, resultado de la preocupación por los impactos 
ambientales de la forma física de las ciudades. Sin embargo, el significado político e ideológico de este discurso también ha estado orientado a legitimar los procesos de urbanización en curso, donde la ciudad sostenible ha pasado a ser un medio de administración técnica y científica de la sociedad y el espacio (Brand, 2001, 2007).

Recientemente, las evidencias y consecuencias del proceso de cambio climático han propiciado el paso de la figura de la ciudad sostenible a la de la ciudad resiliente. Los organismos internacionales han incorporado y difundido la resiliencia urbana como parte de la necesidad de articular la gestión ambiental con la gestión del riesgo, ante las nuevas dinámicas de desastres de gran magnitud y la vulnerabilidad urbana. Como lo señalan Metzger y Robert (2013), ante la imposibilidad de eliminar los riesgos y las limitaciones de las políticas de prevención, se ha dado paso a las políticas de preparación ante desastres, con el consecuente éxito del concepto de la resiliencia urbana. La figura de la ciudad resiliente está vinculada a la valoración positiva de la idea de crisis urbana, la cual resulta incluso deseable para el fortalecimiento y la evolución en la perspectiva de los sistemas complejos. En esta aproximación, centrada en los peligros de los riesgos, la visión de exterioridad de los fenómenos queda reforzada por el enfoque local de los problemas y de las soluciones (Metzger \& Robert, 2013).

\subsubsection{Riesgo e incertidumbre en las reglas y principios del urbanismo}

La crítica al urbanismo y la planificación urbana tradicional, desde su enfoque operacional, ha estado dirigida a la capacidad limitada para prever y programar el futuro a partir de sus instrumentos y procesos. La proyección comprensiva de un futuro a largo plazo, realizada a través de los instrumentos tradicionales de planificación urbana, tuvo que enfrentarse a tres grandes dificultades: la complejidad sistémica de las ciudades, la diversidad socioespacial y de agentes urbanos, y la incertidumbre frente a la posibilidad de un futuro (no) planeado, lo que desembocó en la crisis de la planificación urbana en la década de 1970 (Fernández Güell, 2006).

Esto ha dado paso a la incorporación de la figura de la estrategia en la planificación, mediante un giro del paradigma de la planificación del desarrollo hacia el paradigma de gobernanza neoliberal (de Mattos, 2010). Este enfoque, particularmente técnico y con instrumentos analíticos extraídos de la práctica empresarial, se soportan por un tridente de retos económicos, ambientales y sociales que son abordados desde estrategias de competitividad, sostenibilidad y equidad. De esta forma, el ideal del desarrollo, con base en un fuerte intervencionismo estatal, ha sido sustituido por el crecimiento económico sustentado en el libre juego de las fuerzas de mercado y la reducción de la intervención a partir de la figura de la gobernanza (de Mattos, 2010; Fernández Güell, 2006).

Desde una perspectiva alternativa, la idea de un urbanismo reflexivo reconoce las nuevas condiciones establecidas por el proceso de la modernización reflexiva. A diferencia del modelo metodológico económico y empresarial de la planificación estratégica, el urbanismo reflexivo respondería al reconocimiento de otras racionalidades. Significa la capacidad de cuestionar y tomar decisiones dirigidas a la acción política, donde los contenidos sociales justifican las decisiones políticas. No existiría necesariamente una continuidad de un modelo-método, como el planteado desde la planificación estratégica, sino que existiría también la posibilidad de cuestionar y cambiar ese método. El urbanismo se entendería entonces como un proceso conflictivo y dialéctico en el que se gestionan intereses económicos y sociales contradictorios y valores culturales diversos, donde el consenso no sería el punto de partida del proyecto urbano sino el de llegada (Borja, 2009). 


\section{LA INSTITUCIONALIZACIÓN DEL RIESGO EN BOGOTÁ Y COLOMBIA}

Colombia ha sido un país que se ha situado a la vanguardia en los procesos de implementación de políticas y programas para la atención, prevención y gestión del riesgo y los desastres en Latinoamérica, en una historia marcada por la ocurrencia periódica de desastres y la reacción social a estos eventos, obligando a los distintos gobiernos nacionales y locales a realizar programas específicos de atención y reconstrucción y a realizar ajustes en sus políticas de atención y gestión de riesgos, desastres y emergencias.

En este sentido, la institucionalización del riesgo en Colombia tuvo su origen en la tradición de la respuesta de emergencia, dominante durante gran parte del siglo XX hasta la década de 1980, la cual orientaba la acción institucional a la atención inmediata de situaciones consideradas imprevisibles o de origen natural.

El viraje hacia la formación de una política de gestión del riesgo si inició desde mediados de la década de 1980 (Zeiderman \& Ramírez Elizalde, 2010) con la formulación e implementación del Sistema Nacional para la Prevención y Atención de Desastres -SNPAD-, actualmente Sistema Nacional para la Gestión del Riesgo de Desastres -SNGRD-. Las tragedias de Armero y de la toma del Palacio de Justicia, ocurridos el mes de noviembre de 1985, provocaron la reacción e indignación popular, consolidando un ambiente de crisis de gobernabilidad en el gobierno del Presidente Belisario Betancur (1982-1986), lo que obligó al gobierno entrante del Presidente Virgilio Barco (1986-1990) a revisar el esquema de atención de desastres (Ramírez Gómez \& Cardona Arboleda, 1996). Esto llevó a la adopción del enfoque de la prevención de desastres, en el que se reconoce la existencia de una dimensión social que permite adoptar acciones de prevención y preparación para evitar o reducir los efectos de las amenazas naturales (Ramírez Gómez \& Cardona Arboleda, 1996; Zeiderman \& Ramírez Elizalde, 2010).

En la década de 1990 este enfoque se amplió a la gestión del riesgo de desastres a partir de la agenda internacional impulsada de la ONU, la cual definió los desastres como situaciones que amenazan el desarrollo de la sociedad, por lo cual su gestión debía ser incorporada en los ejercicios de la planificación del desarrollo y la planificación territorial (Naciones Unidas, 2001). La mitigación y prevención de desastres se incorporaron así como componentes del proceso de desarrollo socioeconómico del país y el Estado, por lo que se tuvieron que establecer "los mecanismos institucionales para planificar y gobernar el territorio nacional de acuerdo con evaluaciones y cálculos de riesgo" (Zeiderman \& Ramírez Elizalde, 2010, p. 125). Esta política nacional, vigente en la actualidad, ha estado orientada a gestionar fundamentalmente aquellos riesgos que ya han tomado una forma material, donde la atención de emergencias, la prevención de desastres y la gestión de riesgos son expresiones de una misma tradición de gobierno técnico sobre el territorio y la ciudad

\section{$\frac{\text { Amenaza } \times \text { Vulnerabilidad } x \text { Exposición }}{\text { Resiliencia o capacidad de afrontamiento }}=$ Riesgo de desastre}

Figura 4. Definición del riesgo de desastre.

Fuente: Estrategia Internacional para la Reducción de Desastres, Naciones Unidas.

En el caso específico de Bogotá se presentan algunas variaciones con base en el reconocimiento de la dimensión social del riesgo y la vulnerabilidad y el reconocimiento de su expresión diferencial sobre el territorio (Figura 5). En este sentido, el enfoque de la gestión social del riesgo ha comenzado a consolidar particularmente a partir de la administración distrital de Petro (2012-2015), en la cual se articularon e integraron los riesgos considerados de origen natural con aquellos considerados de origen social. Esto implicó un aumento en la formulación y ejecución de programas y proyectos urbanos integrales para la gestión social del riesgo a nivel local.

A pesar de este desarrollo particular, en la tradición institucionalizada en Colombia el riesgo se ha convertido fundamentalmente en un problema técnico de gestión de las amenazas de origen natural o geofísicas y de la exposición material frente a dichas amenazas. Desde esta perspectiva, la aproximación territorial y urbana al riesgo de desastres ha adquirido forma esencialmente mediante los ejercicios de zonificación del suelo, que son incorporados como parte de las cartografías de los Planes de Ordenamiento Territorial -POT- y los programas de reubicación que de allí se derivan. La posibilidad de desarrollar una gestión prospectiva del riesgo (de aquellos riesgos que aún no existen) en la que se evite en un primer lugar la configuración de las situaciones de desastre, se presenta como un objetivo aún distante (Lavell \& Maskrey, 2013). Esto explica por qué en el contexto colombiano la visión del desastre y su atención y prevención, fundamentalmente desde enfoques técnicos, ha mantenido el dominio sobre la visión social y política del riesgo como proceso moderno reflexivo. 




Figura 5. Evolución de la institucionalización del riesgo en Colombia. Fuente: elaboración propia.

\section{CIUDAD Y RIESGO EN LA CUENCA URBANA DEL RÍO TUNJUELO}

En el caso del territorio del Tunjuelo en Bogotá, la unidad de análisis de la cuenca adquiere una relevancia particular al ser el río el elemento histórico de articulación y desarticulación de la ciudad, en una relación conflictiva que ha definido el carácter complejo del proceso de urbanización de la cuenca y su materialización en la forma y el espacio urbano actual. De esta forma, el río Tunjuelo ha adquirido tres cualidades que se expresan de manera simultánea y desarticulada: 1 . Es un borde o límite urbano desde la perspectiva de la administración local y la planeación zonal, 2. Es un eje de vertebración ambiental sobre el que se estructura el territorio a nivel físico-espacial, y 3. Es un elemento urbano anodino en relación con el tejido socio-económico de la ciudad, dada su apropiación y privatización por parte de las industrias extractivas allí localizadas.

En este sentido, la cuenca del río Tunjuelo expone de manera dramática los procesos espaciales asociados a la formación de una espacialidad del riesgo, los cuales han tomado fuerza en los últimos años en el contexto local. Se trata de una pieza fundamental en el desarrollo urbano de los barrios populares, obreros y de origen informal en la ciudad de Bogotá. En la actualidad su territorio alberga más de 2,5 millones de habitantes de grupos socioeconómicos de ingresos bajos y medios-bajos, quienes configuraron, junto con la acción de los urbanizadores y parceladores, un territorio urbano al margen de los ejercicios control y planeación por parte de la administración de la ciudad. Aquí, la acción e intervención del Estado ha estado orientada sobre la base de la acción no planeada y la respuesta inmediata ante situaciones críticas una vez éstas ya se han configurado o una vez la presión social de los habitantes de la cuenca ha exigido la actuación pública. Esta pasividad en la iniciativa pública ha permitido que la cuenca urbana del río Tunjuelo se haya constituido en el cinturón de pobreza más extenso de Colombia, equivalente en tamaño y población a la ciudad de Medellín.

A manera de síntesis, la cuenca urbana del río Tunjuelo se caracteriza por tener un carácter socioespacial marcadamente homogéneo y complejo, definido por los procesos de urbanización de los barrios obreros, populares y de origen informal, sobre un espacio ecológicamente frágil y socialmente diferenciado con respecto a las otras cuencas de la ciudad. El área de influencia de la cuenca a nivel de unidades de planeación comprende el 32\% del suelo urbano de Bogotá (12.272 Ha., de las cuales $9.994 \mathrm{Ha}$. corresponden directamente a la cuenca del Tunjuelo), alberga el 34\% del total de la población (2'500 mil habitantes), el $57 \%$ de los habitantes de viviendas de estratos bajos (2'150 mil habitantes, en las clasificaciones 0,1 y 2) y el $50 \%$ del déficit cuantitativo de vivienda de la ciudad (95 mil unidades) para el año 2011. Adicionalmente, se traslapan allí actividades de alto impacto, relacionadas con economías de extracción (1.140 Ha. del área urbana), junto con el 46\% de la Estructura Ecológica Principal del área urbana de Bogotá (2.565 Ha.). 




Fuente: elaboración propia a partir del Mapa de Referencia de la IDECA (junio de 2015).

\subsection{La estructuración y reestructuración espacial de la cuenca}

Una lectura histórica del proceso de urbanización permite identificar cuatro grandes procesos urbanos que han definido la forma socioespacial de la cuenca y que se expresan de manera simultánea en la actualidad. Los primeros dos procesos, sobre los que se definió la estructuración espacial de la cuenca a partir de la década de 1930, han respondido en su origen a las lógicas de la necesidad, producto de las dinámicas propias del desarrollo urbano asociadas a los problemas del acceso al suelo, la vivienda y los servicios básicos. Los segundos dos procesos, sobre los que se está definiendo la reestructuración espacial actualmente en curso, responden a la lógica de la contingencia y son producto de los efectos del desarrollo urbano expresados en la acumulación y liberación periódica de riesgos y desastres. En este contexto, ante la magnitud y complejidad del proceso de urbanización, la acción planeada ha quedado relegada a un segundo plano o ha sido insuficiente en la definición de la dinámica y la forma urbana (Figura 9). 




El proceso de urbanización y estructuración espacial de la cuenca del río Tunjuelo se inició en la década de 1940, alcanzando en un periodo de 75 años una población de más de 2'500 mil habitantes en 12.000 Ha de suelo urbanizado (Figura 10). Sin embargo, el río Tunjuelo y su cuenca se incorporaron funcionalmente a la ciudad de Bogotá en la década de 1930, al construirse la ampliación del sistema de acueducto de la ciudad, en una lógica mediada por las dinámicas de extracción de los recursos naturales allí existentes, particularmente asociados al abastecimiento de agua y de materiales de construcción como arenas y arcillas.

El proceso de urbanización iniciado en 1940 se caracterizó por la ausencia del Estado y la solidaridad comunitaria. Si bien el río y la cuenca definieron el espacio geográfico sobre el que se instaló el tejido urbano, fue la unidad barrial el espacio de intercambio y estructuración del tejido social de los nuevos pobladores. La precariedad material en torno a la construcción del barrio, en sus ámbitos públicos y privados, exigió la consolidación de redes de solidaridad comunitaria para resolver las necesidades derivadas de la ausencia de servicios públicos y la construcción de la vivienda. Adicionalmente, la distancia con respecto a la ciudad reforzaría la necesidad de consolidar los tejidos sociales y las dinámicas económicas a escala barrial (Zambrano Pantoja, 2004).

A diferencia del primer periodo de expansión de la ciudad (1930-1950), que respondió fundamentalmente a procesos de desplazamiento intraurbano, durante las siguientes décadas (1960-1990) la dinámica de expansión incluyó de manera importante la población migrante de otras regiones del país. Esta migración, sumada a la mejora en las condiciones de sanidad y reducción de las tasas de mortalidad de los habitantes de Bogotá, explica el estallido urbano que tuvo como protagonista a la cuenca del río Tunjuelo, receptora de los grupos de trabajadores de bajos ingresos. En este contexto y ante los altos costos y la imposibilidad de los programas masivos de vivienda para dar solución habitacional al conjunto de la población de bajos ingresos, el proceso de racionalización de los recursos económicos y las políticas en vivienda terminarían por posicionar las políticas orientadas hacia el cumplimiento de normas mínimas y la oferta de lotes con servicios, como mecanismo para contrarrestar el mercado ilegal de tierras de la ciudad (Salazar Ferro, 2007; Zambrano Pantoja, 2004).

Para la década de 1980 la acción pública se concentró en la "definición y adecuación permanente de fórmulas para definir el monto y la forma de otorgar los subsidios de vivienda y [...] en la regulación de la acción constructora, a través de la aplicación de normas urbanísticas municipales a los proyectos "elegibles» para subsidio" (Salazar Ferro, 2007, p. 202). Finalmente, en las últimas dos décadas del siglo XX, la acción pública se limitó a la definición de códigos urbanos, situación que cambiaría con la expedición y puesta en marcha de la ley 388 de 1997. Esta Ley significó la adopción de un nuevo marco legal, institucional y técnico para la planeación territorial, bajo el cual se exigiría a los municipios la formulación de los POT, lo cual estuvo asociado a la definición de proyectos y programas públicos, así como las regulaciones necesarias para llevar a cabo un modelo territorial a largo plazo. Sin embargo, al momento en 
que es aprobada la Ley 388 de 1997 y la posterior adopción del primer POT de Bogotá en el año 2000, la forma socioespacial de la cuenca urbana del río Tunjuelo habría alcanzado ya su pleno desarrollo. La explosión de la ciudad informal cerraría su ciclo y tomarían relevancia en los procesos de estructuración espacial de la cuenca del Tunjuelo los desarrollos formales en torno al modelo de Metrovivienda, organizado bajo la figura de un banco de tierras (Salazar Ferro, 2007).
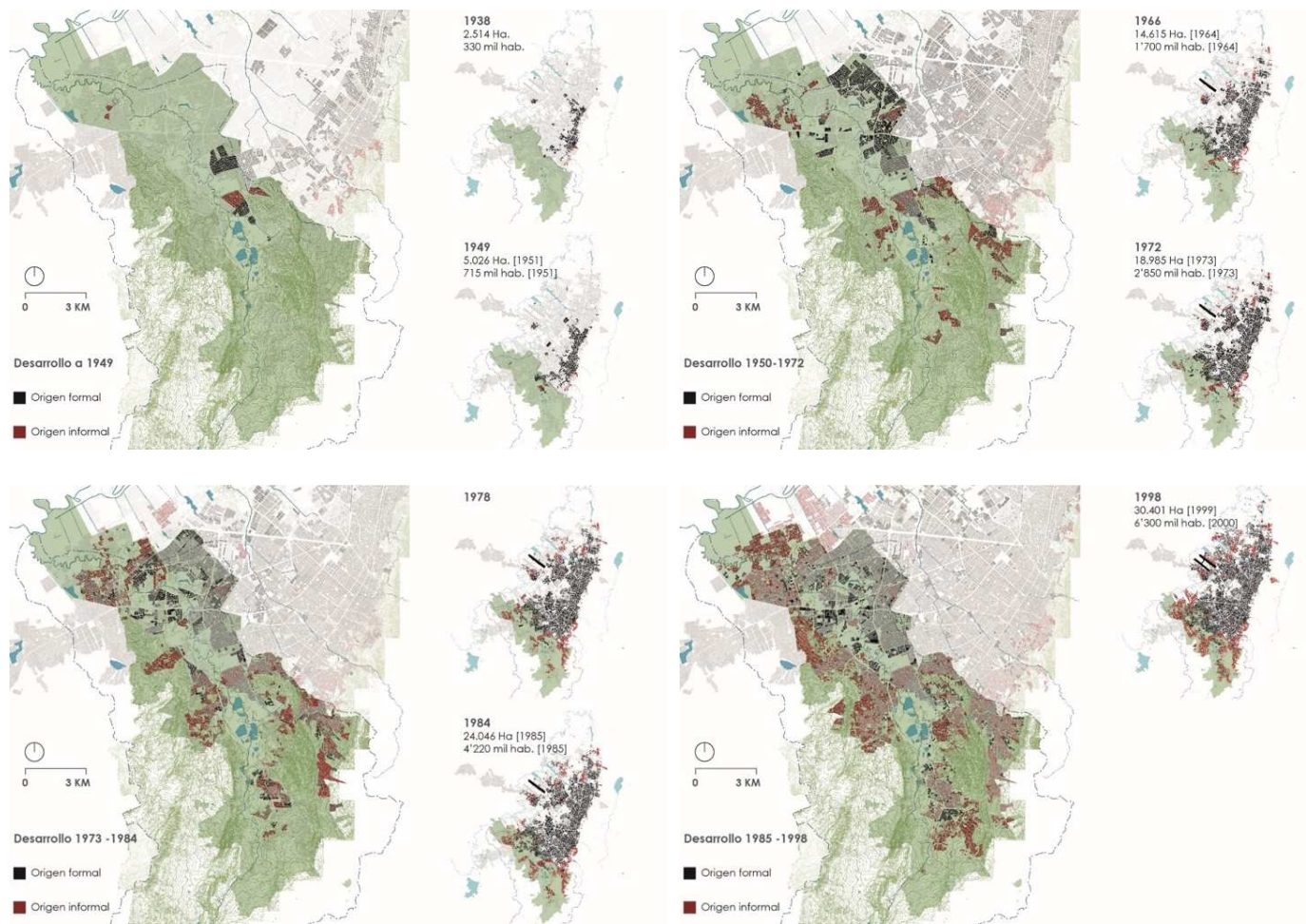

Figura 10. Desarrollo urbano en la cuenca del río Tunjuelo 1949-1998

Fuente: elaboración propia a partir de cartografía "Formas de crecimiento urbano", Servicio WMS Ortofoto de la ciudad de Bogotá, D.C. Año 1998, Universidad Nacional; Boletín "Población y desarrollo urbano", SDP (2010) y Series de Población 1985-2020, DANE.

\subsection{Producción, acumulación y distribución desigual de riesgos}

Si la estructuración espacial de la cuenca ha sido el resultado de procesos urbanos asociados a la lógica de la necesidad por el acceso al suelo, la vivienda y los servicios básicos, la reestructuración espacial actualmente en curso es el resultado de un proceso histórico de urbanización diferencial que se manifiesta en una distribución desigual de daños y pérdidas por situaciones de emergencias y desastres en Bogotá y por una distribución desigual de riesgos. Históricamente el territorio de la cuenca ha sido depositario de las acciones contingentes para dar solución a las crisis urbanas asociadas, entre otros factores, al abastecimiento de agua, la oferta de suelo y vivienda, los desastres y las acciones de reubicación o la disposición de basuras. Está dinámica ha dado como resultado una espacialidad en la que las situaciones de segregación, marginalidad y desigualdad social y económica de los habitantes de la cuenca se combina con una espacialidad del riesgo en proceso de formación. 
Eventos reportados



Relación Tunjuelo/Bogotá

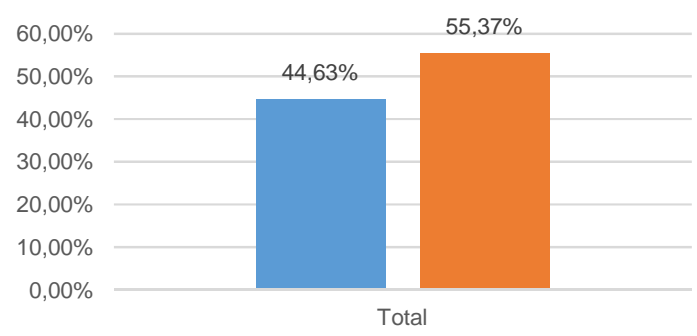

ETunjuelo $\quad$ Resto de Bogotá

Figura 11. Remoción, inundación y encharcamientos Bogotá 2002-2015

Fuente: elaboración propia a partir de registro de emergencias, calamidades y desastres, ODGR-CC.



\% de población expuesta sobre la población total de Bogotá



Figura 12. Población expuesta a alto riesgo Tunjuelo/Bogotá 2005-2015

Fuente: elaboración propia a partir de exposición frente a amenazas, ODGR-CC.

En este sentido, la cuenca del Tunjuelo concentra el $44 \%$ de los eventos de inundación y remoción en masa reportados en Bogotá en el periodo 2002-2015 (Figura 11). De la misma manera, concentra el 47,6\% de las áreas urbanas y el $46,5 \%$ de la población expuesta en Bogotá a niveles altos de amenaza por estos mismos fenómenos. Esta distribución desigual, desde una perspectiva histórica, ha respondido a un proceso sistémico de distribución desigual de riesgos antes que ser el producto de decisiones o eventos de carácter aislado. En este sentido, en el periodo 2005-2015 la participación de la población expuesta a alto riesgo con respecto a la población total de la cuenca ha pasado de un $9,33 \%$ a un $10,14 \%$, lo cual representa una tasa de crecimiento de población en alto riesgo del 2,21\% anual, superior a la tasa de crecimiento global de población en la cuenca, que se encuentra en el 1,36\% para el mismo periodo. En este sentido y de acuerdo a los datos obtenidos, se está presentando un fenómeno de desarrollo urbano y concentración de población en zonas de alto riesgo que van a tener que estar sujetas en un futuro mediato a programas de mitigación y reubicación o que directamente se van a ver afectadas por situaciones de desastre, absorbiendo de manera desigual los efectos del modelo de urbanización de la ciudad. 


\subsection{La estrategia urbana vs la espacialidad del riesgo}

La estrategia urbana y espacial para la consolidación del modelo territorial a largo plazo, contemplada en la modificación del POT de 2003, buscaba materializarse con la implementación de una serie de Operaciones Estratégicas para la constitución de una red de centralidades en la ciudad, la cual no fue implementada. En este sentido, ha sido la lógica de la contingencia y los procesos de distribución desigual de riesgos, daños y pérdidas, los que han definido la reestructuración espacial de la cuenca en los últimos 15 años. Frente a estos procesos, la práctica urbanística se ha limitado fundamentalmente a la zonificación y al desarrollo creciente de medidas y programas para la mitigación de los efectos de las amenazas naturales, que no afectan las relaciones desiguales de riesgo, y que tienen un carácter pasivo y marginal frente a la posibilidad de proyectar una estrategia territorial de carácter integral.

Inversión absoluta

(Correspondiente a compromisos)


Figura 14. Inversión en vivienda, riesgo y desarrollos informales 1998-2015 Fuente: elaboración propia a partir de Memorias de Planes de Desarrollo Distritales, SDP.

Inversión absoluta

(Correspondiente a compromisos)
Participación sobre inversión total del periodo (Correspondiente a compromisos)


\$ Valores indexados a 2016

Figura 15. Inversión en reasentamientos y recuperación de suelo 1998-2015

Fuente: elaboración propia a partir de Memorias de Planes de Desarrollo Distritales, SDP.

Este proceso de reestructuración se puede evidenciar con el incremento significativo, desde el año 1998, de las inversiones asociadas al riesgo versus la reducción progresiva de las inversiones orientadas a suplir el déficit de infraestructura básica (Figura 14). Estas inversiones se han orientado fundamentalmente a la protección y recuperación de suelo y al reasentamiento de familias afectadas por exposición alta a amenazas naturales (Figura 15). En este sentido, si bien en el POT se calculó y proyectó en su momento la reubicación de 14.200 familias para el año 2010, en la actualidad esta proyección de familias a reubicar se 
mantiene en aumento, alcanzando para el año 2015 un acumulado en reubicación y proyección de 16.000 familias (Figura 16). Adicionalmente, se ha presentado un aumento significativo de la inversión pública para la adecuación de suelo afectado por amenazas naturales en los últimos dos periodos administrativos, donde se han intervenido $94 \mathrm{Ha}$. con una inversión de 255 mil millones de pesos, frente a una disminución del suelo habilitado para la urbanización, que ha alcanzado en este mismo periodo $130 \mathrm{Ha}$. e inversiones por 310 mil millones de pesos (Figura 17).

De esta forma, en la medida en que se ha incrementado la magnitud y complejidad de las acciones urbanas asociadas al riesgo, en particular en cuanto a los procesos de reasentamiento y adecuación de los suelos liberados, se ha requerido la formulación de nuevos modelos de intervención con un alcance integral y estructural, aunque por fuera de la estrategia territorial definida en el POT. Estas respuestas, aun limitadas, han integrado los programas de mejoramiento de barrios con la formulación de proyectos urbanos integrales para la gestión del riesgo, como los observados bajo la figura de las API de 2015 (Figura 18 y 19) o en el Plan de Mejoramiento Urbanístico de altos de la Estancia de 2013 (Figura 20).

Familias reasentadas por periodo



..... Familias por reasentar

—— Subsidios de vivienda
Acumulado de familias reasentadas

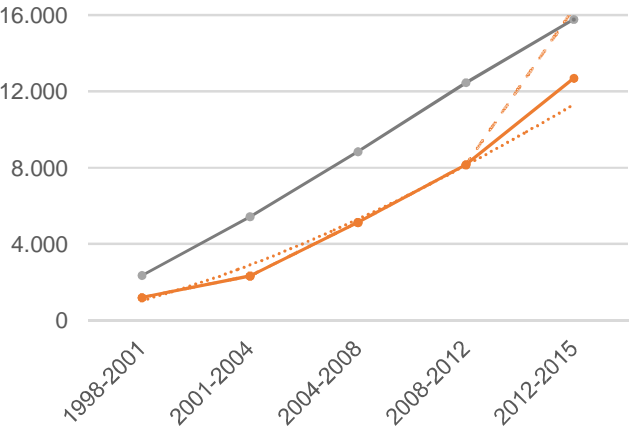

- - Familias por reasentar — Familias reasentadas

Familias recomendadas

En 2008 se estimaban en 23.000 el total de familias a reasentar según CVP.

Figura 16. Familias reasentadas y subsidios política de vivienda Distrital 1998-2015

Fuente: elaboración propia a partir de Memorias de Planes de Desarrollo Distritales, SDP.

Ha. adecuadas por riesgo vs Ha. habilitadas para nuevos desarrollos



Inversión en protección y recuperación de suelo por riesgo vs inversión en gestión de suelo para nuevos desarrollos



$\$$ Valores indexados a 2016

Figura 17. Gestión, protección y recuperación de suelo urbano 1998-2015

Fuente: elaboración propia a partir de Memorias de Planes de Desarrollo Distritales, SDP.

En este sentido, la estrategia urbana visualizada en el POT ha sido sustituida en la práctica urbanística por toda una serie de intervenciones desarticuladas de carácter estructural sobre el territorio, fundamentadas en un primer momento sobre la visión del desastre y por los enfoques técnicos de la gestión del riesgo, y más 
recientemente enriquecidas por el enfoque de la gestión social del riesgo y la formulación de proyectos urbanos integrales asociados, configurando una nueva espacialidad del riesgo en el territorio del Tunjuelo.



Figura 18. Modelo de intervención del PMIB - 2011

Fuente: SDH, Programa de Mejoramiento Integral de Barrios, 2015.
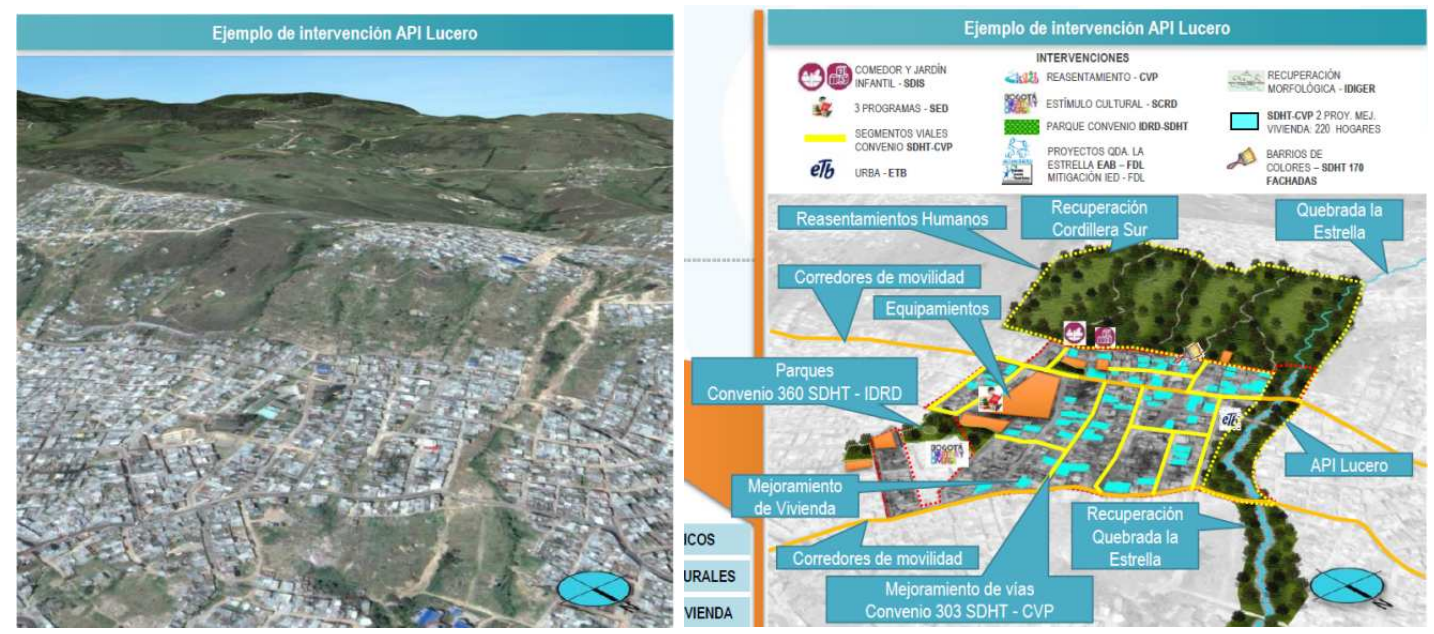

Figura 19. Ejemplo de propuesta de intervención API Lucero Fuente: SDH, El espacio público en el mejoramiento integral, 2015.

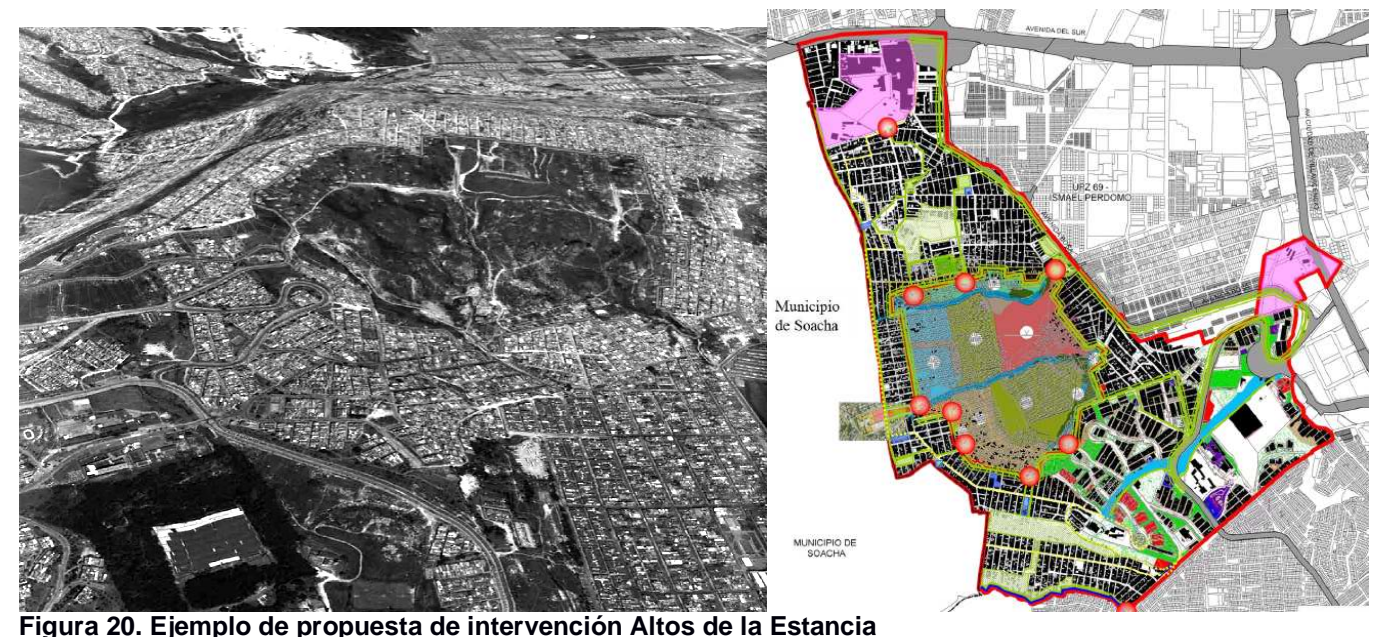

Figura 20. Ejemplo de propuesta de intervención Altos de la Estancia Fuente: SDP, Plan de mejoramiento urbanístico Altos de la Estancia, 2013. 


\section{CONSIDERACIONES FINALES ANTE LA EMERGENCIA DE UNA ESPACIALIDAD DEL RIESGO}

El riesgo es un componente activo en los procesos de configuración socioespacial en Bogotá en la actualidad. Aproximarse a la comprensión de los procesos urbanos y territoriales contemporáneos, implica necesariamente estudiar las relaciones de riesgo, asociadas a procesos históricos en su producción, acumulación y distribución desigual. En la medida que los enfoques técnicos dominan las aproximaciones y debates frente al riesgo, este conocimiento se convierte en un dispositivo para la administración técnica del territorio con poca incidencia o posibilidad de participación social democrática, por lo cual es necesario tener en cuenta dos consideraciones:

a) Las aproximaciones técnicas, centradas en la expresión material de las amenazas naturales, son insuficientes o no son adecuadas para dar cuenta de la complejidad dinámica del riesgo y sus impactos en la producción social del espacio.

b) Los procesos asociados al riesgo tienen impactos de orden estructural sobre los tejidos urbanos de la ciudad, por lo cual es conveniente ampliar la participación ciudadana informada en torno a la toma de decisiones que cambiarán drásticamente las condiciones de vida de quienes habitan los espacios de riesgo.

Dada la función pública y social del urbanismo, se debería privilegiar la construcción de consensos para el ordenamiento, la planeación y la gestión de la ciudad. Es necesario así que en los procesos de investigación sobre ciudad y riesgo se aborden enfoques permitan ampliar las discusiones técnicas y especializadas en las que se centran actualmente estos debates. Esto permitiría a académicos, profesionales y políticos no especializados o no familiarizados con las aproximaciones técnicas, así como al conjunto de la población, acceder a un conocimiento determinante para la administración del territorio. En este sentido, se plantea la necesidad de trabajar en dos sentidos: 1. En la formulación de nuevas estrategias urbanas que incorporen el riesgo como parte de un urbanismo reflexivo, y 2 . En el desarrollo de nuevos instrumentos de planeación y gestión urbana que respondan a las exigencias de la espacialidad del riesgo en formación.

\subsection{La formulación de estrategias urbanas asociadas a un urbanismo reflexivo}

Los procesos urbanos asociados al riesgo tienen impactos de orden estructural sobre los tejidos urbanos de la ciudad. En el caso específico de la investigación adelantada, se pudo establecer que estos procesos pusieron en marcha y están definiendo la reestructuración espacial de la cuenca urbana del río Tunjuelo a partir de una lógica de la contingencia que se está desarrollando al margen de la definición de una estrategia urbana o territorial orientada a la generación de un consenso social. Esta lógica es el resultado de la liberación continua de riesgos, producidos y acumulados históricamente, los cuales configuran situaciones de desastre que tienen que ser intervenidos desde la iniciativa pública bien sea antes o después de su ocurrencia.

Reconocer e incorporar dentro de la formulación de las estrategias urbanas la espacialidad del riesgo, específica, diferenciada y contingente, es necesario para orientar los procesos de reestructuración espacial de la ciudad en función de la definición de un proyecto urbano común e incluyente. Si bien esta estrategia requeriría necesariamente la proyección de intervenciones de orden técnico y estructural sobre el territorio para la mitigación y adaptación de situaciones de riesgo de desastre que ya se hayan configurado, también requeriría la concepción de acciones orientadas a la equilibrar los procesos de distribución desigual de riesgos en la ciudad y la cuenca. Desde la perspectiva de su distribución desigual, el riesgo deja de ser fundamentalmente un problema de administración técnica y pasa a ser un problema social y político.

En este sentido, el primer proceso productor de riesgos en la ciudad es la urbanización misma, más incluso cuando se trata de desarrollos en contextos de origen informal. Un urbanismo reflexivo implica cuestionar la perspectiva desarrollista de la expansión urbana o los macroproyectos de vivienda, más aun cuando éstos están dirigidos a población vulnerable. Por otra parte, las acciones estructurales sobre el territorio para la gestión del riesgo de desastre y las relacionadas con procesos de relocalización deberían estar orientadas a romper con los procesos de asunción desigual de riesgos por parte de la población afectada, evitando reproducir desde la acción institucional los procesos de reestructuración espacial asociados a la lógica de la contingencia. 


\subsection{Sobre los instrumentos de planeación y gestión urbana}

Es necesario reconocer y dar continuidad a la concepción de instrumentos de planeación y gestión urbana que responden a una aproximación social e integral al riesgo. Estos instrumentos no deben estar centrados exclusivamente en la concepción y desarrollo de acciones de mitigación y adaptación frente a situaciones de exposición a amenazas naturales, ya que estas acciones no garantizan la ruptura de la dinámica de reproducción de situaciones contingentes. Para esto, se requiere profundizar en la concepción de proyectos urbanos integrales que abarquen las espacialidades del riesgo más allá de las áreas directamente afectadas por amenazas y que supongan la participación de los habitantes locales en la concepción de los espacios intervenidos y transformados. Frente a esto, se proponen dos aproximaciones para la participación de la población en los procesos de reestructuración espacial:

a) La redefinición del carácter de los suelos protegidos y recuperados por procesos de gestión del riesgo. Estos suelos en la actualidad adquieren la categoría de suelos de protección por riesgo con impactos negativos en la vida urbana de los territorios afectados, lo que limita el acceso y la participación de los habitantes (tanto de quienes se mantienen como de quienes se reubican) en su administración social y colectiva. En este sentido, parte de las reivindicaciones sociales que se rastrearon en el desarrollo de la investigación están orientadas a la exigencia de una administración social de los territorios transformados, al desarrollo de acciones de reivindicación de la identidad y la memoria y a la implementación de actividades productivas de bajo impacto, entre otras. Sería necesario profundizar a futuro en la definición de nuevas categorías de suelo urbano que pudieran responder a esta espacialidad de manera más acertada y menos restrictiva.

b) La participación en la gestión social de los recursos públicos orientados al riesgo: los recursos orientados a la gestión del riesgo vienen incrementándose de manera significativa en los últimos años, en parte por el aumento en la configuración de situaciones de riesgo y desastre, como por la formación de una conciencia social y política frente al tema. Es importante que los habitantes de estos contextos encuentren instrumentos para la gestión social de los recursos orientados a atender las situaciones de riesgo y la exposición a amenazas, en la perspectiva de ampliar la concepción del riesgo más allá de la aproximación dominante centrada en la dimensión material de las amenazas. Esto permitiría, por una parte, que el recurso orientado a la gestión del riesgo no sea acaparado por unas pocas empresas especializadas en su gestión técnica, y por otra, que los habitantes de los espacios de riesgo pasen de ser concebidos como afectados, a los que se les asigna un rol pasivo, a que sean concebidos como ciudadanos, con un rol activo en la toma de decisiones con respecto al territorio, los recursos y la transformación de estas espacialidades.

Finalmente, si bien el riesgo puede entenderse como un problema urbano complejo en términos técnicos y políticos, ofrece la posibilidad de otorgarle al espacio urbano una nueva plasticidad, que en la actualidad está acaparada por la pragmática económica de la inversión privada y el capital financiero. Sí se entiende en los términos del carácter dual de los procesos globales de reestructuración urbana, la espacialidad del riesgo entendida desde una perspectiva social y política, permitiría reposicionar la dimensión de la utopía en el espacio urbano, ya que exigiría pensar creativa y socialmente el espacio. Por el contrario, desde una perspectiva exclusivamente técnica, esta espacialidad pierde el potencial utópico, quedando reducida a un ejercicio pragmático de administración técnica del territorio.

\section{BIBLIOGRAFÍA}

Ascher, F. (2004). Los nuevos principios del urbanismo. (M. Hernández Díaz, Trad.). Madrid, España: Alianza.

Beck, U. (1998). La sociedad del riesgo. Hacia una nueva modernidad. (J. Navarro, D. Jiménez, \& M. R. Borrás, Trads.). Barcelona, España: Paidos.



Beriain, J. (2007). El doble sentido de las consecuencias perversas de la modernidad [prólogo]. En C. Sánchez Capdequí (Trad.), Las consecuencias perversas de la modernidad: Modernidad, contingencia y riesgo (2. ${ }^{\text {a }}$ ed., pp. 7-29). Barcelona, España: Anthropos.

Borja, J. (2009). Luces y sombras del urbanismo de Barcelona. Barcelona, España: UOC. 
Borja, J. (2013). Revolución urbana y derechos ciudadanos. Madrid, España: Alianza.

Brand, P. C. (2001). La construcción ambiental del bienestar urbano: Caso de Medellín, Colombia. Economía, Sociedad y Territorio, 3(9), 1-24.

Cárdenas Roa, L. A. (2016). Riesgo y reestructuración espacial en la cuenca urbana del río Tunjuelo (Maestría). Universidad Nacional de Colombia. Facultad de Artes, Bogotá.

Choay, F. (1997). The Rule and the Model. On the Theory of Architecture and Urbanism. (D. Bratton, Ed.). Cambridge, Massachusetts: The MIT Press.

Davis, M. (2007). Planeta de ciudades miseria. (J. M. Amoroto Salido, Trad.). Madrid, España: Foca.

Fernández Güell, J. M. (2006). Planificación estratégica de ciudades. Nuevos instrumentos y procesos (Nueva edición revisada y aumentada). Barcelona, España: Reverté.

Sassen, S. (2015). Expulsiones. Brutalidad y complejidad en la economía global. Buenos Aires, Argentina: Katz.

Zambrano Pantoja, F. (2004). Historia de la Localidad de Tunjuelito: El Poblamiento del Valle Medio del Río Tunjuelo. Bogotá, Colombia: Guadalupe.

\section{FUENTES ELECTRÓNICAS}

Brand, P. C. (2007). Green Subjection: The Politics of Neoliberal Urban Environmental Management. International Journal of Urban and Regional Research, 31(3), 616-632. https://doi.org/10.1111/j.14682427.2007.00748.x (Consulta: 18/02/2015)

de Mattos, C. A. (2010). Una nueva geografía latinoamericana en el tránsito de la planificación a la gobernanza, del desarrollo al crecimiento. EURE-Revista Latinoamericana de Estudios Urbanos Regionales, 36(108), 167-179. https://doi.org/10.4067/S0250-71612010000200010 (Consulta: 27/11/2014)

D’Ercole, R., Hardy, S., Metzger, P., \& Jérémy, R. (Eds.). (2009). Vulnerabilidades urbanas en los países andinos: Introducción general. Bulletin de l'Institut français d'Études Andines, 38(3), 401-410.

https://doi.org/10.4000/bifea.2216 (Consulta: 26/02/2015)

La RED. (1993). Los desastres no son naturales. http://www.desenredando.org/public/libros/1993/ldnsn/ (Consulta: 11/02/2015)

La RED. (1996a). Ciudades en riesgo: Degradación ambiental, riesgos urbanos y desastres. La RED / Usaid. http://www.inpe.br/crs/geodesastres/conteudo/livros/LaRed_1996_Ciudades_en_riesgos.pdf (Consulta: 04/02/2014)

La RED. (1996b). Estado, sociedad y gestión de los desastres en América Latina: En busca del paradigma perdido. La RED / Flacso / ITDG-Perú. http://www.desenredando.org/public/libros/1996/esyg/esyg_Intro_dic18-2002.pdf (Consulta: 11/02/2015)

Lampis, A. (2013). Ciudad y resiliencia: Una cuestión al estilo de Escher. Territorios, (28), 9-19. http://www.redalyc.org/articulo.oa?id=35728173001 (Consulta: 12/02/2015)

Lampis, A., \& Rubiano, L. (2012). ¡Y siguen culpando a la lluvia!: Vulnerabilidad ambiental y social en el Altos de la Estancia, Bogotá, Colombia. En La RED, Perspectivas de investigación y acción frente al cambio climático en Latinoamérica (pp. 177-220). Mérida, Venezuela: La RED / Cigir. http://www.desenredando.org/public/2012/LaRed_Desastres_y_Sociedad_2012-07_web.pdf (Consulta: 30/01/2014)

Lavell, A. (2010). Gestión Ambiental y Gestión del Riesgo de Desastre en el Contexto del Cambio Climático: Una Aproximación al Desarrollo de un Concepto y Definición Integral para Dirigir la Intervención a través de un Plan Nacional de Desarrollo (No. DNP MO-084-2010). Departamento Nacional de Planeación [DNP]. http://www.desenredando.org/public/2013/2010-09- 
26_DNP_Lavell_DocumentoConceptual_GestionDelRiesgo.pdf (Consulta: 06/02/2014)

García Acosta, V. (2005). El riesgo como construcción social y la construcción social de riesgos. Desacatos, (19), 11-24. http://www.redalyc.org/articulo.oa?id=13901902 (Consulta: 26/02/2015)

Lavell, A., \& Maskrey, A. (2013). The Future of Disaster Risk Management: An On-going Discussion. San José, Costa Rica: Flacso / Unisdr. http://www.redalyc.org/articulo.oa?id=13901902 (Consulta: 11/02/2015)

Metzger, P., \& Robert, J. (2013). Elementos de reflexión sobre la resiliencia urbana: Usos criticables y aportes potenciales. Territorios, (28), 21-40. http://www.redalyc.org/articulo.oa?id=13901902 (Consulta: $06 / 03 / 2015)$

Naciones Unidas. (2001). Marco de Acción para la implementación de la Estrategia Internacional para la Reducción de los Desastres (EIRD). http://www.redalyc.org/articulo.oa?id=13901902 (Consulta: 24/02/2016)

Ramírez Gómez, F., \& Cardona Arboleda, O. (1996). El sistema nacional para la atención y prevención de desastres en Colombia. En Estado, sociedad y gestión de los desastres en América Latina: en busca del paradigma perdido. (pp. 214-255). Lima, Perú: La RED / Flacso / ITDG-Perú. http://www.redalyc.org/articulo.oa?id=13901902 (Consulta: 28/09/2014)

Rebotier, J. (2009). La dimensión territorial del riesgo urbano en Caracas: Características y alcances. Trace, (56), 11-25. http://www.redalyc.org/articulo.oa?id=13901902 (Consulta: 26/01/2014)

Rebotier, J. (2014). La vulnerabilidad urbana: entre reducción de riesgo y emancipación social. Ejemplos en Venezuela. Polis, 13(38), 573-595. https://doi.org/10.4067/S0718-65682014000200025 (Consulta: 22/03/2015)

Salazar Ferro, J. F. (2007). El proyecto urbano en la acción pública de vivienda en los años 60 y 70 en Colombia. En J. C. Del Castillo Daza (Ed.), Áreas residenciales en Bogotá (pp. 198-203). Bogotá, Colombia: Universidad Nacional de Colombia. http://www.redalyc.org/articulo.oa?id=13901902 (Consulta: 25/04/2016)

Zeiderman, A. (2012). On shaky ground: The making of risk in Bogotá. Environment \& Planning, 44(7), 15701588. https://doi.org/10.1068/a44283 (Consulta: 25/02/2015)

Zeiderman, A., \& Ramírez Elizalde, L. A. (2010). Apocalipsis anunciado: Un viraje en la política de riesgo en Colombia a partir de 1985. Revista de Ingeniería, (31), 119-131.

http://www.redalyc.org/articulo.oa?id=13901902 (Consulta: 05/09/2014) 